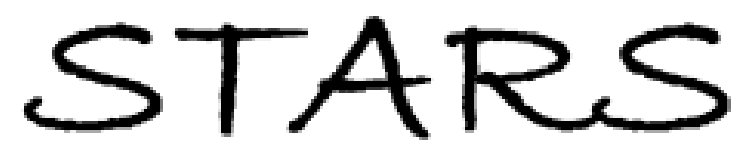

University of Central Florida

STARS

$1-1-2008$

\title{
On the phase identification of dc magnetron sputtered Pt-Ru alloy thin films
}

\author{
A. P. Warren \\ University of Central Florida \\ R. M. Todi \\ University of Central Florida \\ B. Yao \\ University of Central Florida \\ K. Barmak \\ K. B. Sundaram \\ University of Central Florida
}

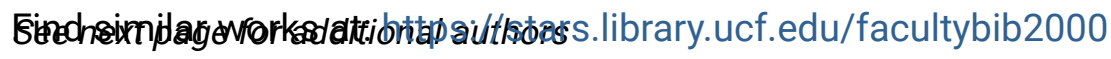

University of Central Florida Libraries http://library.ucf.edu

This Article is brought to you for free and open access by the Faculty Bibliography at STARS. It has been accepted for inclusion in Faculty Bibliography 2000 s by an authorized administrator of STARS. For more information, please contactSTARS@ucf.edu.

\section{Recommended Citation}

Warren, A. P.; Todi, R. M.; Yao, B.; Barmak, K.; Sundaram, K. B.; and Coffey, K. R., "On the phase identification of dc magnetron sputtered Pt-Ru alloy thin films" (2008). Faculty Bibliography 2000 s. 1128. https://stars.library.ucf.edu/facultybib2000/1128

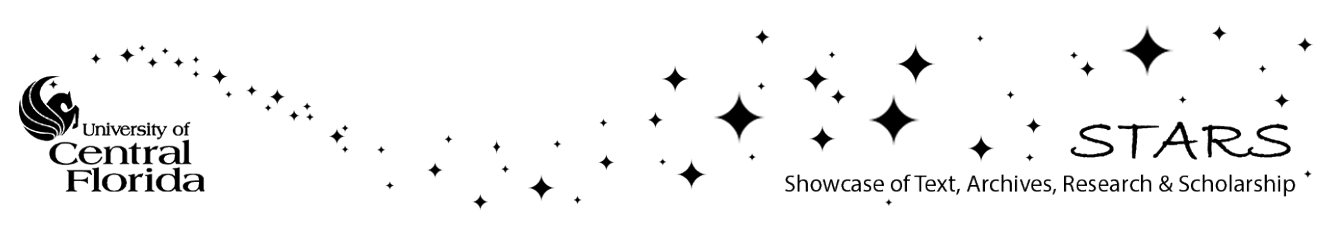




\section{Authors}

A. P. Warren, R. M. Todi, B. Yao, K. Barmak, K. B. Sundaram, and K. R. Coffey 
On the phase identification of dc magnetron sputtered Pt-Ru alloy thin films

A. P. Warren, R. M. Todi, B. Yao, K. Barmak, K. B. Sundaram, and K. R. Coffey

Citation: Journal of Vacuum Science \& Technology A 26, 1208 (2008); doi: 10.1116/1.2966422

View online: https://doi.org/10.1116/1.2966422

View Table of Contents: https://avs.scitation.org/toc/jva/26/5

Published by the American Vacuum Society

\section{ARTICLES YOU MAY BE INTERESTED IN}

Comparison of the agglomeration behavior of thin metallic films on $\mathrm{SiO}_{2}$ Journal of Vacuum Science \& Technology A 23, 1152 (2005); https://doi.org/10.1116/1.1861943

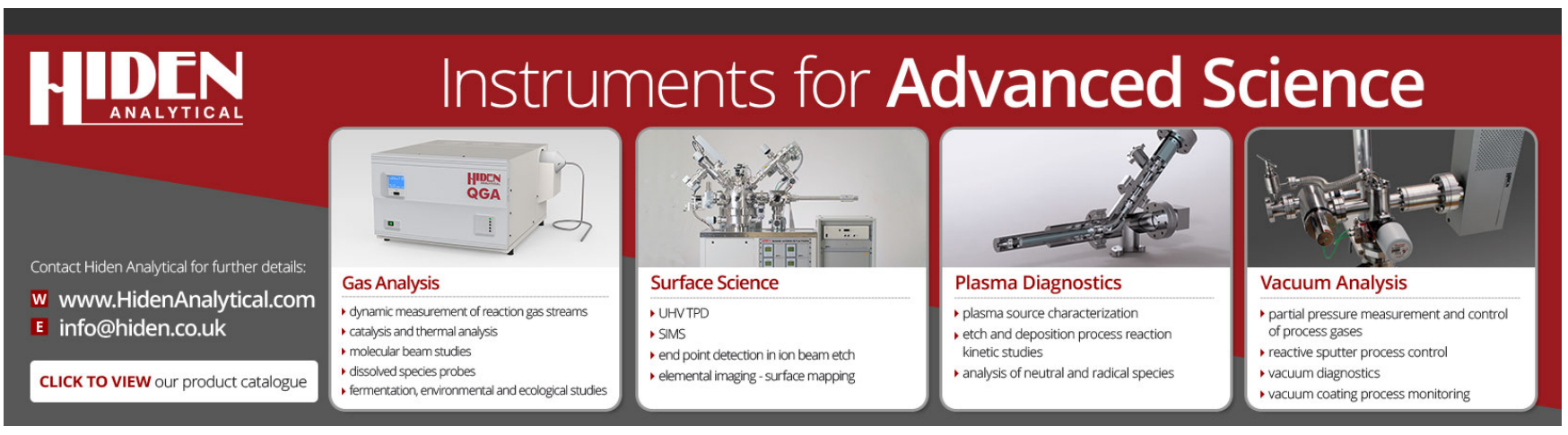




\title{
On the phase identification of dc magnetron sputtered Pt-Ru alloy thin films
}

\author{
A. P. Warren ${ }^{\text {a) }}$ \\ Advanced Materials Processing and Analysis Center, University of Central Florida, 4000 Central Florida \\ Blvd., Orlando, Florida 32816 \\ R. M. Todi \\ Advanced Materials Processing and Analysis Center, University of Central Florida, 4000 Central Florida \\ Blvd., Orlando, Florida 32816 and School of Electrical Engineering and Computer Science, \\ University of Central Florida, 4000 Central Florida Blvd., Orlando, Florida 32816 \\ B. Yao \\ Advanced Materials Processing and Analysis Center, University of Central Florida, 4000 Central Florida \\ Blvd., Orlando, Florida 32816 \\ K. Barmak \\ Department of Materials Science and Engineering, Carnegie Mellon University, 5000 Forbes Ave., \\ Pittsburgh, Pennsylvania 15213 \\ K. B. Sundaram \\ Advanced Materials Processing and Analysis Center, University of Central Florida, 4000 Central Florida \\ Blvd., Orlando, Florida 32816 and School of Electrical Engineering and Computer Science, \\ University of Central Florida, 4000 Central Florida Blvd., Orlando, Florida 32816
}

K. R. Coffey

Advanced Materials Processing and Analysis Center, University of Central Florida, 4000 Central Florida Blvd., Orlando, Florida 32816

(Received 27 February 2008; accepted 7 July 2008; published 6 August 2008)

\begin{abstract}
The microstructure and the electronic work function of Pt-Ru alloy thin films spanning the compositional range from pure $\mathrm{Pt}$ to pure $\mathrm{Ru}$ were investigated. Nominally $50 \mathrm{~nm}$ thick films were cosputtered from elemental targets in an ultrahigh vacuum chamber. X-ray reflectivity and Rutherford backscattering spectroscopy were used to determine the film thicknesses and compositions. The electronic work function of the alloy film samples was determined by analysis of the capacitance-voltage characteristics of films deposited as part of a metal-oxide-semiconductor capacitor structure and found to range from $4.8 \mathrm{eV}$ for pure $\mathrm{Ru}$ to $5.2 \mathrm{eV}$ for pure Pt. To better understand the variation in work function for the intermediate compositions, the films were characterized by transmission electron microscopy and x-ray and electron diffractions. A notable increase in the compositional range of the hexagonal close packed (hcp) phase was observed, suggesting a metastable extension of the hcp phase stability as compared to bulk Pt-Ru alloys. The steepest change in the electronic work function for the intermediate alloy compositions coincided with a rapid change in the $c / a$ ratio of the hcp phase. () 2008 American Vacuum Society.
\end{abstract}

[DOI: $10.1116 / 1.2966422$ ]

\section{INTRODUCTION}

The continued scaling of device dimensions in complementary metal-oxide-semiconductor (MOS) technology in the sub-70 $\mathrm{nm}$ region requires an alternative high dielectric (high- $\kappa$ ) oxide layer to counter tunneling leakage currents and a metallic gate electrode to address limitations of polysilicon. ${ }^{1}$ As the technology scaling continues for higher density and performance, limitations associated with the polysilicon gate become increasingly important. These limitations include increasing polydepletion effects, high gate resistance, boron penetration, and compatibility issues with high permittivity gate dielectric films., ${ }^{2,3}$ Metal gates can potentially address these limitations and are therefore attracting

\footnotetext{
a) Tel.: 407-882-1516; electronic mail: awarren@mail.ucf.edu
}

research interest. ${ }^{4,5}$ Several approaches, including implanted metals, ${ }^{6}$ fully silicided gates, ${ }^{7}$ and metal alloys, ${ }^{8}$ have been investigated in an effort to achieve tunable work function metal gates. Recently, the use of metal bilayers has been reported as another method to achieve the desired work function $^{9-11}$ where the work function is continuously changed from that of one metal to the other.

A key parameter for the choice of the metal gate material is its work function. For $p$-channel MOS ( $p$-MOS), Pt and $\mathrm{Ru}$ are both possible choices due to their higher work function value. Alloys of these metals can also be considered to achieve an optimized or "tunable" work function. Replacing the polysilicon in the gate typically requires a dual metal approach; a metal with a $p$-MOS work function and a second metal with $n$-MOS work function. The work function $\left(\Phi_{m}\right)$ of the metal must be near the conduction band edge for 
$n$-MOS and near valence band edge of silicon for $p$-MOS in order to satisfy the low power and low supply voltage demand. A detailed study of the resulting phases for a binary metal alloy system is important, as it can be expected to impact the important properties of the gate electrode material, such as its work function, electrical resistivity, and thermal stability. Relatively little work has been done to investigate work function in two-phase alloys. However, for fcc materials, the dependence of work function and lattice parameter on alloy composition has been demonstrated theoretically and corroborated experimentally. ${ }^{12}$ Furthermore, the effect of composition and phase transformation on work function in Ta-Si alloy films has also been investigated. ${ }^{13}$ In this paper we report a detailed phase characterization of PtRu binary-alloy thin films performed predominantly by $\mathrm{x}$-ray diffraction (XRD).

\section{EXPERIMENT}

$\mathrm{Pt}-\mathrm{Ru}$ alloys were prepared by sputter deposition in an ultrahigh vacuum system. The alloys were formed by cosputtering $\mathrm{Pt}$ and $\mathrm{Ru}$ from elemental targets with a purity of $99.95 \%$. A quartz crystal thickness monitor was used to calibrate deposition rates at various dc power settings which allowed for alloy composition to be controlled. The base pressure was $\sim 1 \times 10^{-8}$ Torr, while the deposition pressure was maintained at 4 mTorr with a flow of 20 SCCM (SSCM denotes cubic centimeter per minute at STP) of Ar. The purity of the Ar process gas was maintained by a hot reactive metal getter. Process gas contamination in the deposition chamber at the typical deposition pressure of 4 mTorr was confirmed to be less than $10 \mathrm{ppm}$ (the instrumental sensitivity limit) by closed ion source quadrapole mass spectrometry.

To extract the work function of the binary alloys of Pt$\mathrm{Ru}, \mathrm{MOS}$-capacitor structures were fabricated. Aluminum was thermally evaporated, in a cryo filament evaporator, on the back side of the silicon wafer and annealed in Ar ambient at $500{ }^{\circ} \mathrm{C}$ for $30 \mathrm{~min}$ to establish an Ohmic contact. The metal alloys were deposited after back side $\mathrm{Al} \mathrm{Ohmic} \mathrm{con-}$ tacts were established. HP 4192 impedance analyzer was used to measure high-frequency $C-V$ data at $1 \mathrm{MHz}$. The flatband voltage $V_{\mathrm{fb}}$ was extracted from the $C$ - $V$ characteristics based on the North Carolina State University $C-V$ program $^{14}$ and plotted against the effective oxide thickness to determine the work function for the Pt-Ru binary-alloy system. The work function was also determined from the Schottky-barrier height, extracted from the $I-V$ and $C-V$ characteristics of the Schottky-barrier diode. It is well known that the Schottky-barrier height depends on the quality of the semiconductor material, the method of surface preparation prior to metallization, and the metallization itself. The $n$-type silicon wafers were cleaned, and the back side aluminum Ohmic contact was first established as described above. The silicon surface was hydrogen terminated by hydrofluoric acid just before introducing the wafer in the vacuum chamber for sputter deposition of Pt-Ru alloy metals to form the Schottky diode. I-V characteristics of the Pt-Ru Schottky contacts were obtained under dark conditions, at room temperature

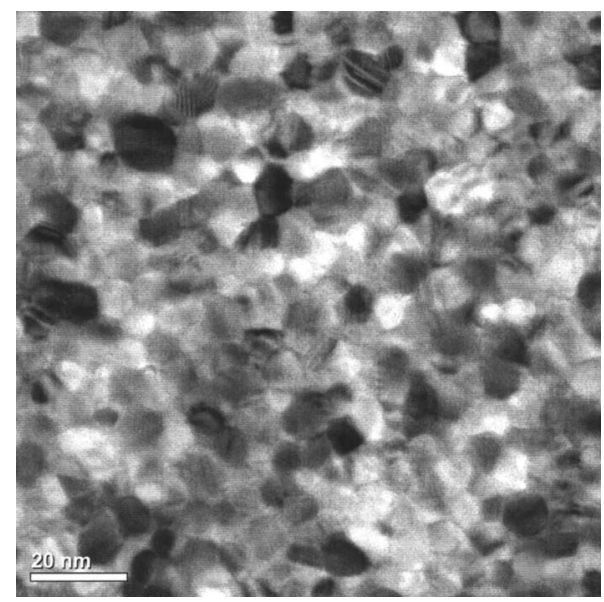

FIG. 1. Bright field TEM micrograph of Pt-Ru alloy thin film with a Ru concentration of 84 at. \%. The film is polycrystalline and continuous with a grain size on the order of $100 \AA$.

(295 K), using Keithley Instruments picoammeter/voltage source model 6487. A Keithley Instruments $C-V$ analyzer model 590 was used to measure the $C$ - $V$ characteristics of the Pt-Ru Schottky contacts, and the barrier height was extracted to calculate the metal work function. ${ }^{10,11}$

Films were deposited onto glass substrates for x-ray diffraction analysis to prevent the artifacts that often result from multiple scattering events within a single crystal Si substrate. Films for transmission electron microscopy were deposited on oxidized Si wafer substrates.

Rutherford backscattering spectroscopy (RBS), performed with a General IONIX 1.7 MV Tandetron RBS system, was used to determine alloy composition, and the x-ray characterization was performed on a Rigaku D-Max system using $\mathrm{Cu} K \alpha$ radiation. X-ray reflectivity was used to determine film thickness by analyzing specular reflectivity patterns with BEDE REFS software. ${ }^{15}$ Thin film XRD was used to investigate the crystal structure of the alloys as a function of their respective compositions. Analysis of XRD patterns was performed with JADE 7.5 from MDI. The Pt-Ru thin films, on glass substrates, were cut into approximately $1 \times 1 \mathrm{~cm}^{2}$ squares and were rinsed with alcohol to provide a relatively clean surface prior to XRD characterization.

\section{RESULTS}

Bright field transmission electron microscopy (TEM) imaging reveals the films to be polycrystalline and continuous with a negligible void fraction (Fig. 1). There is some variation in grain size between samples that we attribute to small variations in film thickness.

The structural dependence on composition can be seen in the XRD patterns and is qualitatively confirmed with electron diffraction. Figure 2 shows grazing incidence XRD of thin films spanning the compositional range from pure $\mathrm{Pt}$ to pure $\mathrm{Ru}$ and intensities have been offset for clarity. The pure Pt and pure Ru samples are clearly single-phase face centered cubic (fcc) and hexagonal close packed (hcp), respectively, and what is of interest is the transition from fcc to hcp 

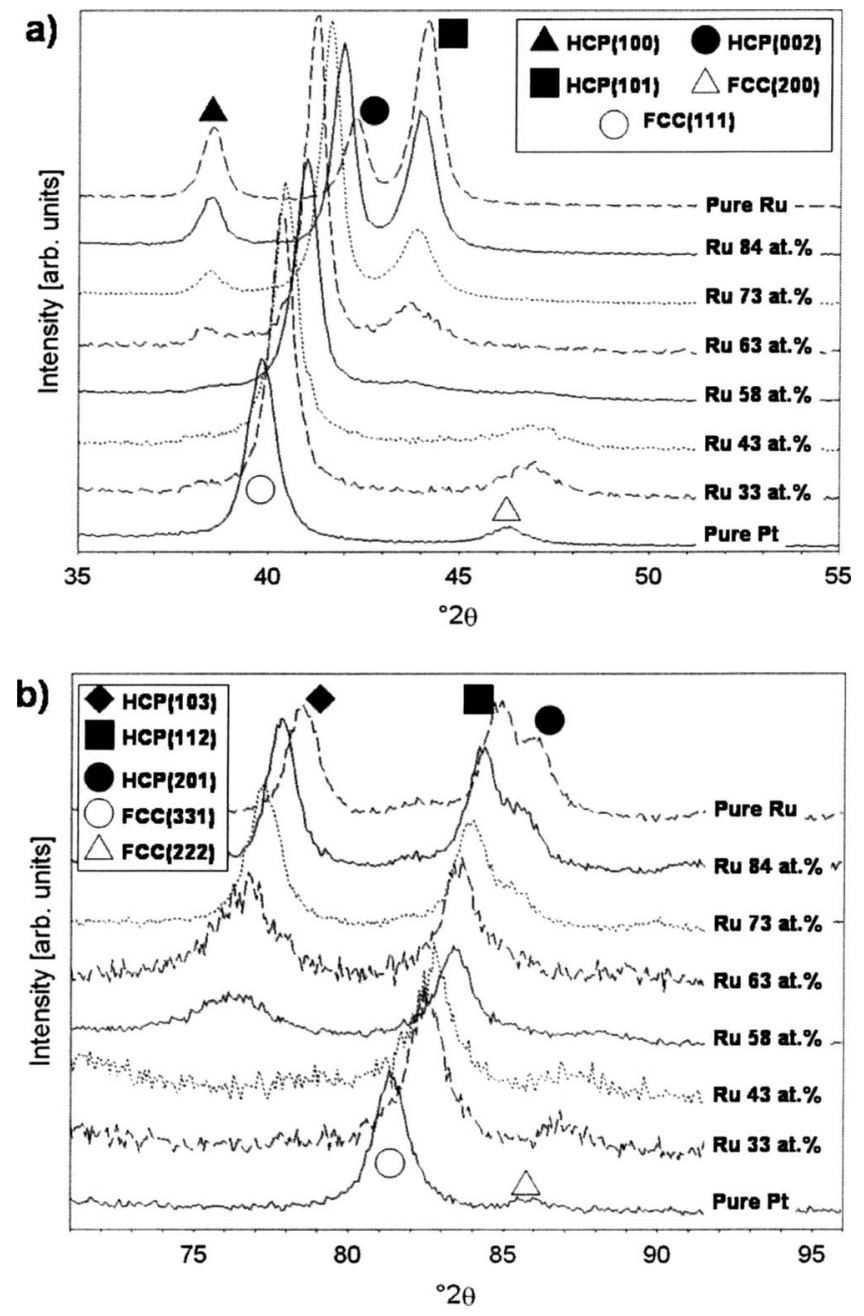

FIG. 2. XRD patterns of (a) low angle and (b) high angle reflections of $\mathrm{Pt}-\mathrm{Ru}$ alloys spanning the compositional range from pure $\mathrm{Pt}$ to pure $\mathrm{Ru}$. (a) The fcc(111) peak shifts to higher angles and is replaced by the hcp(002) peak. A decreasing fcc(200) peak intensity and increasing hcp(100) and (101) peak intensities are seen. (b) The hcp(103) peak emerges with increasing Ru concentration. Also, the fcc(331) peak shifts to higher angles and splits into hcp(112) and (201) peaks.

that occurs at intermediate compositions. The low angle reflections [Fig. 2(a)] reveal a fcc(111) peak for pure Pt that gradually shifts to higher angles and can ultimately be indexed as the hcp(002) peak in pure Ru. As this peak is common to both crystal structures, it cannot be used as evidence of the absence of either one of these two phases. However, the $\mathrm{fcc}(200)$ peak is unique to the fcc phase and it is observed to decrease in intensity with increasing Ru concentration and is not discernible in Fig. 2(a) above 43 at. \% Ru. This observation is accompanied by the growth of the hcp(100) and (101) peaks with increasing Ru concentration. Both of these peaks are clearly present in the sample having 63 at. \% Ru, and some indication of the hcp(101) peak is also present at 58 at. \% Ru. The higher angle XRD peaks for these same samples [Fig. 2(b)] clearly show the presence of the hcp phase at 58 at. $\% \mathrm{Ru}$, as the (103) peak is unique to the hcp phase. The hcp(103) peak intensity increases as the $\mathrm{Ru}$ concentration is increased. Also evident is the shift to

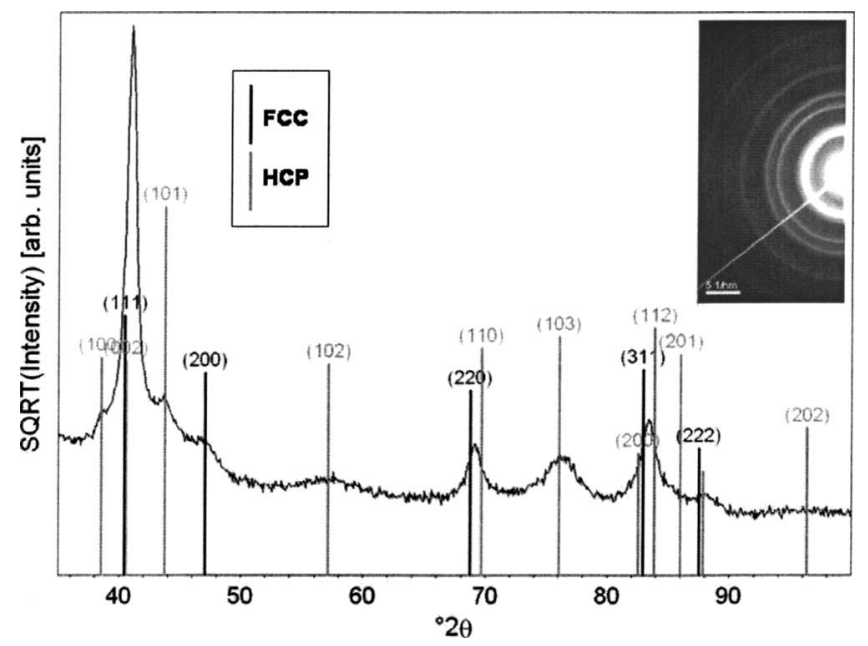

FIG. 3. X-ray and electron diffraction patterns of a Pt-Ru alloy thin film with 58 at. \% Ru are shown. A mixed fcc-hcp phase is evident from the presence of a fcc(200) reflection and $h c p(101)$ and hcp(100) reflections.

higher angles of the fcc(331) and its splitting into hcp(112) and (201). Clearly, the 58 at. \% Ru sample is at or near the transition with composition from fcc phase to hcp phase and warrants a more detailed examination.

Assessment of the sample with a 43 at. \% Ru concentration (figure not shown) reveals asymmetries in the fcc(111) and fcc(200) peaks. We attribute these asymmetries to hcp(100) and hcp(101), respectively, thereby suggesting a dual phase microstructure at this composition that remains predominately fcc. Figure 3 shows the full range of XRD measured for the 58 at. \% Ru sample and also includes a comparable range of electron diffraction intensities for the same sample (Fig. 3 inset). The presence of both fcc and hcp phases is apparent in that the fcc (200) peak remains a dominant feature of the diffraction pattern, while several hcp peaks are also present. This is evident in both $\mathrm{x}$-ray and electron diffraction data. The fcc(200) remains discernible, albeit less intense, for the sample with a 63 at. \% Ru concentration (not shown) indicating some remaining fcc phase. $\mathrm{Pt}-\mathrm{Ru}$ alloys with $\mathrm{Ru}$ concentrations higher than 63 at. \% have only discernible hcp phase peaks in both electron and $\mathrm{x}$-ray diffractions.

The $d$-spacing and lattice parameters for the fcc and hep phases were obtained from the XRD data. Figure 4 shows the shift of the $d$-spacing of the fcc(111) plane and subsequent $\mathrm{hcp}(002)$ plane as a function of Ru concentration. Increasing the $\mathrm{Ru}$ concentration causes the $d$-spacing of the fcc(111) plane to decrease linearly at a rate of $9 \times 10^{-4} \AA /$ at. \% Ru, while the hcp(002) $d$-spacing decreases at a rate of 1.5 $\times 10^{-3} \AA /$ at. \% Ru. In the cases where fcc(111) and hcp(002) peak overlaps prevent a direct measurement of their respective peak positions, the nonoverlapping reflections were used to calculate the lattice parameters for the fcc and hcp phases and subsequently the $d$-spacing values for the fcc(111) and hcp(002). The observed $d$-space decrease with increasing Ru concentration is attributed to the smaller bonding radius of $\mathrm{Ru}$ as compared to Pt. For samples with a 


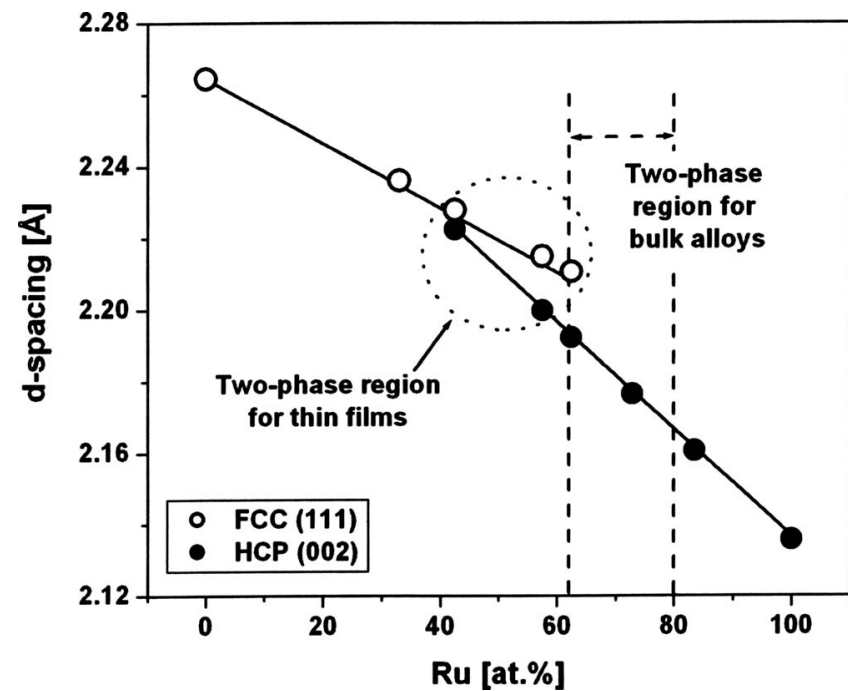

FIG. 4. $d$-spacing extracted from XRD data for the fcc phase (open circles) and the hcp phase (filled circles) is shown as a function of the Ru concentration of the Pt-Ru thin film alloy samples. The two-phase region where both phases are present in the thin films is indicated by the dotted circle. Also shown is the two-phase region expected for bulk alloys (Ref. 16) (dashed lines).

discernible hcp phase, a proportional change in both lattice parameters $(c$ and $a$ ) could not fully account for the measured change in all peak positions, therefore indicating a compositional dependence of the $c / a$ ratio as well. Figure 5 shows the measured $c / a$ ratio as a function of Ru concentration, and the data follow a weakly quadratic trend.

Figure 6 presents the results of measurements of the work function for these alloys as a function of composition, and a complex nonlinear behavior is evident. The variation in work function follows the observed metastable extension of the hcp phase identified by XRD (Fig. 4). Regions of weak compositional dependence are observed in the single-phase and near single-phase regions of the alloy thin films. Steep compositional dependence is observed across the intermediate compositional range where the $c / a$ ratio is observed to de-

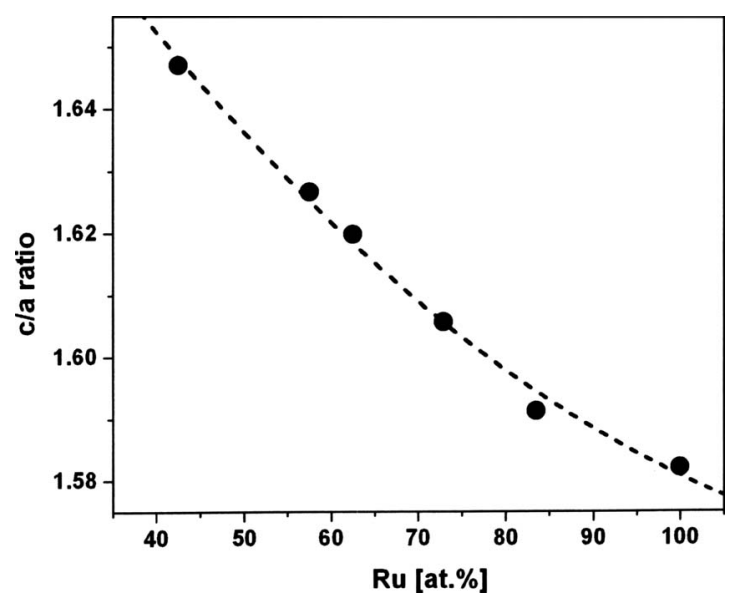

FIG. 5. c / a ratio for the hcp phase of the Pt-Ru alloys thin films is shown as a function of $\mathrm{Ru}$ concentration. A quadratic dependence of the $c / a$ ratio on $\mathrm{Ru}$ concentration is evident.

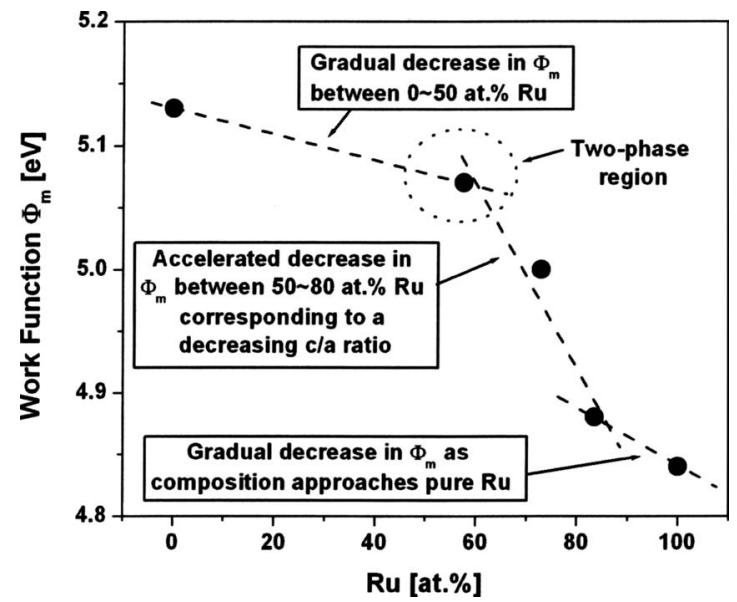

FIG. 6. Electronic work function of the Pt-Ru alloy thin films is shown as a function to $\mathrm{Ru}$ concentration. Regions of weak compositional dependence are observed near the compositional extremes, and a steeper compositional dependence is observed across the intermediate compositional range. The two-phase region identified in Fig. 4 is also indicated.

crease most rapidly as Ru concentration is increased. Figure 7 shows the strong correlation between the electronic work function and $c / a$ ratio of the hcp phase samples, where a linear relationship is observed. In previous works ${ }^{10,11}$ we reported that that the change of film properties with composition is consistent with the two-phase region of the equilibrium phase diagram. This conclusion, although still valid, has been extended and refined: Here we report that this same compositional range consists primarily of a nonequilibrium extension of the hcp phase in which the $c / a$ ratio varies rapidly.

\section{DISCUSSION}

According to the equilibrium phase diagram for bulk $\mathrm{Pt}-\mathrm{Ru}$ alloys, ${ }^{16} \mathrm{a} \mathrm{Ru}$ content of less than 62 at. \% is expected to consist of only fcc phase, and Ru concentrations of more than 80 at. \% are expected to be hcp. Both fcc and hep phases of $\mathrm{Pt}$ and $\mathrm{Ru}$ are expected to be present for $\mathrm{Ru}$ con-

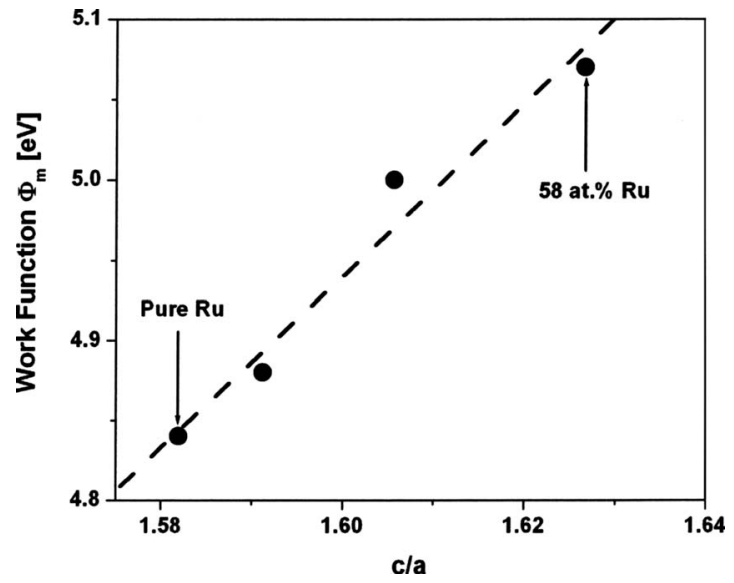

FIG. 7. Approximately linear relationship is observed between the electronic work function and the $c / a$ ratio of the hcp phase in Pt-Ru alloys. 
centrations between 62 and 80 at. \%. However, structural analysis of the Pt-Ru thin film alloy system reveals a notable increase in the compositional range of the hop phase, suggesting a metastable extension of the hcp phase stability as compared to bulk Pt-Ru alloys. In the case of thin films, a mixed hcp-fcc phase becomes apparent with Ru concentrations as low as 43 at. \%. Furthermore, the fcc phase is completely absent (single-phase hcp) for $\mathrm{Ru}$ concentrations higher than approximately 65 at. \%. In addition to the metastable extension of the hcp phase, a gradual change of phase is apparent in the form of a linearly decreasing $d$-space of the fcc(111), and subsequent hcp(002) planes, as Ru concentration is increased. A decrease in $c / a$ ratio is also evident in the hcp phase. When these structural changes with composition are compared to the electronic work function variation, it is evident that the steepest change in work function coincides with the steep change in $c / a$ ratio of the hcp phase.

\section{CONCLUSION}

$\mathrm{PtRu}$ alloy thin films were sputter deposited in an ultrahigh vacuum chamber by dc magnetron sputtering and were extensively characterized by XRD to quantify the phase dependence of the alloy composition in the Pt-Ru alloy thin films. Equilibrium binary phase diagrams ${ }^{15}$ for bulk Pt-Ru alloys indicate a two-phase microstructure for compositions from 62 to 80 at. \% Ru. However, for these thin films a notable increase in the compositional range of the hcp phase was observed, suggesting a metastable extension of the hcp phase stability as compared to bulk Pt-Ru alloys. The steepest change in the electronic work function for the intermediate alloy compositions coincided with a rapid change in the $c / a$ ratio of the hcp phase.

\section{ACKNOWLEDGMENTS}

The authors would like to acknowledge the Materials Characterization Facility at the University of Central Florida and its staff. A.P.W. would also like to acknowledge the IEEE Electron Device Society's masters fellowship program.

${ }^{1}$ G. D. Wilk, R. M. Wallace, and J. M. Anthony, J. Appl. Phys. 89, 5243 (2001).

${ }^{2}$ H. Zhong, S.-N. Hong, Y.-S. Suh, H. Lazar, G. Heuss, and V. Misra, Tech. Dig. - Int. Electron Devices Meet. 2001, 467.

${ }^{3}$ V. Misra, H. Zhong, and H. Lazar, IEEE Electron Device Lett. 23, 354 (2002).

${ }^{4}$ P. Majhi et al., in Proceedings of the 34th European Solid-State Device Research Conference, 21-23 Sept. 2004, pp. 185-188.

${ }^{5}$ C. Cabral, C. Lavoie, A. S. Ozcan, R. S. Amos, V. Narayanan, E. P. Gusev, J. L. Jordan, and J. M. E. Harper, J. Electrochem. Soc. 151, F283 (2004).

${ }^{6}$ R. Lin, Q. Lu, P. Ranade, T.-J. King, and C. Hu, IEEE Electron Device Lett. 23, 49 (2002).

${ }^{7}$ C. Cabral et al., Dig. Tech. Pap. - Symp. VLSI Technol. 2004, 15.

${ }^{8}$ W. Gao, J. F. Conley, and Y. Ono, CMOS Front-end Materials and Processing Technology, MRS Symposia Proceedings No. 765 (Materials Research Society, Pittsburgh, PA, 2003), p. D1.4-1.6.

${ }^{9}$ J. S. Jeon et al., Tech. Dig. - Int. Electron Devices Meet. 2004, 303.

${ }^{10}$ R. M. Todi, A. P. Warren, K. B. Sundaram, K. Barmak, and K. R. Coffey, IEEE Electron Device Lett. 27, 542 (2006).

${ }^{11}$ R. M. Todi, M. S. Erickson, K. B. Sundaram, K. Barmak, and K. R. Coffey, IEEE Trans. Electron Devices 54, 807 (2007).

${ }^{12}$ I. A. Abrikosov and H. L. Skriver, Phys. Rev. B 47, 16532 (1993).

${ }^{13}$ H. Luan et al., Appl. Phys. Lett. 87, 212110 (2005).

${ }^{14}$ J. R. Hauser et al., in Characterization and Metrology for ULSI Technology, AIP Conf. Proc. 449, D. G. Seiler, Ed., pp. 235-239 (1998).

${ }^{15}$ M. Wormington, C. Panaccione, K. M. Matney, and D. K. Bowen, Philos. Trans. R. Soc. London, Ser. A 357, 2827 (1999).

${ }^{16}$ Binary Alloy Phase Diagrams, 2nd ed., edited by T. B. Massalski (ASM International, Materials Park, OH, 1990). 